\title{
Statistical Convergence of Double Sequences of Order $\tilde{\alpha}$
}

\author{
R. Çolak and Y. Altin \\ Department of Mathematics, Firat University, 23119 Elazı̆̆, Turkey \\ Correspondence should be addressed to Y. Altin; yaltin23@yahoo.com
}

Received 14 May 2013; Accepted 10 July 2013

Academic Editor: Dachun Yang

Copyright (C) 2013 R. Çolak and Y. Altin. This is an open access article distributed under the Creative Commons Attribution License, which permits unrestricted use, distribution, and reproduction in any medium, provided the original work is properly cited.

We intend to make a new approach and introduce the concepts of statistical convergence of order $\widetilde{\alpha}$ and strongly $p$-Cesàro summability of order $\widetilde{\alpha}$ for double sequences of complex or real numbers. Also, some relations between the statistical convergence of order $\widetilde{\alpha}$ and strong $p$-Cesàro summability of order $\widetilde{\alpha}$ are given.

\section{Introduction}

The concept of statistical convergence was introduced by Steinhaus [1] and Fast [2] and later investigated by Schoenberg [3] independently for real and complex sequences. Over the years and under different names, statistical convergence has been discussed in the theory of Fourier analysis, ergodic theory, number theory, measure theory, trigonometric series, turnpike theory, and Banach spaces. Later on, it was further investigated from the sequence space point of view and linked with summability theory by Fridy [4], Connor [5], Maddox [6], Rath and Tripathy [7], Šalát [8], Tripathy et al. [9-12], and many others.

The idea of statistical convergence was later extended to double sequences by Mursaleen and Edely [13], Karakaya et al. [14], Móricz [15], and Tripathy et al. [16-18]. More recent developments on double sequences can be found in [19-22].

In the present paper, we introduce and examine the concepts of statistically convergence of double sequences of order $\tilde{\alpha}$ and strong $p$-Cesàro summability of order $\widetilde{\alpha}$ of double sequences of complex or real numbers, where $\tilde{\alpha}$ denotes the pair $(s, t)$, and $s, t$ are any real numbers such that $s, t \in(0,1]$. The order of statistical convergence of a single sequence of numbers was given in [23], and after that, statistical convergence of order $\alpha$ and strong $p$-Cesàro summability of order $\alpha$ were studied by Çolak in [24, 25].

In Section 2, we give a brief information about statistical convergence and strong $p$-Cesàro summability, and we define the concepts of statistical convergence of order $\widetilde{\alpha}$ and strong
$p$-Cesàro summability of order $\widetilde{\alpha}$ and give some results. In Section 3, we give the main results and establish some inclusion relations between $S_{\widetilde{\alpha}}^{2}$ and $w_{p \tilde{\alpha}}^{2}$.

\section{Definitions and Preliminaries}

In this section, mainly we introduce and examine the concepts of the $\tilde{\alpha}$-double density of a subset $E$ of $\mathbb{N} \times \mathbb{N}$, statistical convergence of order $\widetilde{\alpha}$, and strong $p$-Cesàro summability of order $\widetilde{\alpha}$ of the double sequences of complex or real numbers for $\widetilde{\alpha} \in(0,1]$.

$w^{2}$ denotes the space of all double sequences. Let $\ell_{\infty}^{2}$, $c^{2}$ and $c_{0}^{2}$ be the linear spaces of bounded, convergent, and null sequences $x=\left(x_{j k}\right)$ with complex terms, respectively, normed by $\|x\|_{(\infty, 2)}=\sup _{j, k}\left|x_{j k}\right|$, where $j, k \in \mathbb{N}=\{1,2, \ldots\}$ the set of positive integers.

By the convergence of a double sequence, we mean the convergence in Pringsheim's sense [26]. A double sequence $x=\left(x_{j k}\right)$ is said to be convergent in the Pringsheim sense if for every $\varepsilon>0$ there exists $N \in \mathbb{N}$ such that $\left|x_{j k}-L\right|<\varepsilon$ whenever $j, k \geq N$; $L$ is called the Pringsheim limit of $x$.

A double sequence $x=\left(x_{j k}\right)$ is bounded if there exists a positive number $M$ such that $\left|x_{j k}\right| \leq M$ for all $j$ and $k$, that is, if

$$
\|x\|_{(\infty, 2)}=\sup _{j, k}\left|x_{j k}\right|<\infty
$$

Note that in contrast to the case for single sequences, a convergent double sequence need not be bounded. 
Let $K \subseteq \mathbb{N} \times \mathbb{N}$ and let $K(n, m)$ be the number of $(i, j)$ in $K$ such that $i \leq n$ and $j \leq m$. Then, the lower asymptotic density of a set $K \subseteq \mathbb{N} \times \mathbb{N}$ is defined by

$$
\underline{\delta^{2}}(K)=\liminf _{n, m} \frac{K(n, m)}{n m} .
$$

In case the sequence $(K(n, m) / n m)$ has a limit in Pringsheim's sense, then we say that $K$ has a double natural density and is defined by

$$
\delta^{2}(K)=\lim _{n, m} \frac{K(n, m)}{n m} .
$$

Throughout the paper, we take $s, t, u, v \in(0,1]$, otherwise indicated, and for the sake of brevity, we write $\widetilde{\alpha}$ instead of $(s, t)$ and $\widetilde{\beta}$ instead of $(u, v)$. Also, we define

$$
\begin{aligned}
& \tilde{\alpha} \preceq \widetilde{\beta} \Leftrightarrow s \leq u \text { and } t \leq v \\
& \widetilde{\alpha} \prec \widetilde{\beta} \Leftrightarrow s<u \text { and } t<v \\
& \widetilde{\alpha} \cong \widetilde{\beta} \Leftrightarrow s=u \text { and } t=v \\
& \widetilde{\alpha} \in(0,1] \Leftrightarrow s, t \in(0,1] \\
& \widetilde{\beta} \in(0,1] \Leftrightarrow u, v \in(0,1] \\
& \widetilde{\alpha} \cong 1 \text { in case } s=t=1 \\
& \widetilde{\beta} \cong 1 \text { in case } u=v=1 \\
& \widetilde{\alpha}>1 \text { in case } s>1 \text { and } t>1,
\end{aligned}
$$

and furthermore, we write $S_{\widetilde{\alpha}}^{2}$ to denote $S_{(s, t)}^{2}$ and $S_{\widetilde{\beta}}^{2}$ to denote $S_{(u, v)}^{2}$ in the following.

Now we define the $\widetilde{\alpha}$-double density of the set $K \subseteq \mathbb{N} \times \mathbb{N}$ as

$$
\delta_{\tilde{\alpha}}^{2}(K)=\lim _{n, m} \frac{K(n, m)}{n^{s} m^{t}} .
$$

Remark 1. Note that for any set $K \subseteq \mathbb{N} \times \mathbb{N}, \delta_{\tilde{\alpha}}^{2}(K)$ may be greater than 1 , even equal to $\infty$, but $\delta^{2}(K) \leq 1$. Also, $\delta^{2}\left(K^{c}\right)=$ $1-\delta^{2}(K)$ holds but $\delta_{\tilde{\alpha}}^{2}(K)=1-\delta_{\tilde{\alpha}}^{2}(K)$ does not hold in general.

Definition 2. Let $x=\left(x_{j_{k}}\right) \in w^{2}$ and $\widetilde{\alpha} \in(0,1]$ be given. The sequence $\left(x_{j k}\right)$ is said to be statistically convergent of order $\widetilde{\alpha}$ if there is a complex number $\ell$ such that for every $\varepsilon>0$,

$$
\lim _{n \rightarrow \infty} \frac{1}{n^{s} m^{t}}\left|\left\{(j, k): j \leq n, k \leq m:\left|x_{j k}-\ell\right| \geq \varepsilon\right\}\right|=0,
$$

which is the case when we say that $x$ is statistically convergent of order $\widetilde{\alpha}$ to $\ell$. In this case, we write $S_{\widetilde{\alpha}}^{2}-\lim _{j, k} x_{j k}=\ell$ and we denote the set of all statistically convergent double sequences of order $\tilde{\alpha}$ by $S_{\tilde{\alpha}}^{2}$.

In case $\widetilde{\alpha} \cong 1$, the statistical convergence of order $\widetilde{\alpha}$ reduces to the statistical convergence of double sequences [13]. If $x=\left(x_{j k}\right)$ is statistically convergent of order $\tilde{\alpha}$ to the number $\ell$, then $\ell$ is determined uniquely. The statistical convergence of order $\widetilde{\alpha}$ is well defined for $\tilde{\alpha} \in(0,1]$, but it is not well defined for $\tilde{\alpha}>1$. For this, let $x=\left(x_{j k}\right)$ be defined as follows:

$$
x_{j k}= \begin{cases}1, & \text { if } j+k \text { even } \\ 0, & \text { if } j+k \text { odd } .\end{cases}
$$

Then,

$$
\begin{gathered}
\lim _{n, m \rightarrow \infty} \frac{1}{n^{s} m^{t}}\left|\left\{(j, k): j \leq n, k \leq m,\left|x_{j k}-1\right| \geq \varepsilon\right\}\right| \\
\quad \leq \lim _{n, m \rightarrow \infty} \frac{(n / 2+1)(m / 2+1)}{n^{s} m^{t}}=0, \\
\lim _{n, m \rightarrow \infty} \frac{1}{n^{s} m^{t}}\left|\left\{(j, k): j \leq n, k \leq m,\left|x_{j k}\right| \geq \varepsilon\right\}\right| \\
\leq \lim _{n, m \rightarrow \infty} \frac{(n / 2+1)(m / 2+1)}{n^{s} m^{t}}=0
\end{gathered}
$$

for $\widetilde{\alpha}>1$ that is $s>1$ and $t>1$, so that $x=\left(x_{j k}\right)$ statistically converges of order $\tilde{\alpha}$ both to 1 and 0 , that is, $S_{\tilde{\alpha}}^{2}-\lim x_{j k}=1$ and $S_{\tilde{\alpha}}^{2}-\lim x_{j k}=0$. However, this is impossible.

Theorem 3. Let $\tilde{\alpha} \in(0,1]$ and $x=\left(x_{j k}\right), y=\left(y_{j k}\right)$ be sequences of complex numbers. Then,

(i) If $S_{\tilde{\alpha}}^{2}-\lim x_{j k}=x_{0}$ and $c \in \mathbb{C}$, then $S_{\tilde{\alpha}}^{2}-\lim c x_{j k}=c x_{0}$

(ii) If $S_{\tilde{\alpha}}^{2}-\lim x_{j k}=x_{o}$ and $S_{\tilde{\alpha}}^{2}-\lim y_{j k}=y_{0}$, then $S_{\tilde{\alpha}}^{2}-$ $\lim \left(x_{j k}+y_{j k}\right)=x_{0}+y_{0}$.

Proof. (i) is clear in case $c=0$. Suppose that $c \neq 0$; then the proof of (i) follows from

$$
\begin{aligned}
& \frac{1}{n^{s} m^{t}}\left|\left\{(j, k): j \leq n, k \leq m,\left|c x_{j k}-c x_{0}\right| \geq \varepsilon\right\}\right| \\
& \quad=\frac{1}{n^{s} m^{t}}\left|\left\{(j, k): j \leq n, k \leq m,\left|x_{j k}-x_{0}\right| \geq \frac{\varepsilon}{|c|}\right\}\right|
\end{aligned}
$$

and that of (ii) follows from the following inequality:

$$
\begin{aligned}
\frac{1}{n^{s} m^{t}} \mid & \{(j, k): j \leq n, k \leq m, \\
& \left.\quad\left|x_{j k}+y_{j k}-\left(x_{0}+y_{0}\right)\right| \geq \varepsilon\right\} \mid \\
\leq & \frac{1}{n^{s} m^{t}}\left|\left\{(j, k): j \leq n, k \leq m,\left|x_{j k}-x_{0}\right| \geq \frac{\varepsilon}{2}\right\}\right| \\
& +\frac{1}{n^{s} m^{t}}\left|\left\{(j, k): j \leq n, k \leq m,\left|y_{j k}-y_{0}\right| \geq \frac{\varepsilon}{2}\right\}\right| .
\end{aligned}
$$

It is easy to see that every convergent double sequence is statistically convergent of order $\tilde{\alpha}$ to the same number, that is $c^{2} \subset S_{\widetilde{\alpha}}^{2}$ for each $\widetilde{\alpha} \in(0,1]$ that is for each pair of $(s, t)$ such that $s, t \in(0,1]$. However, the converse does not hold. For example, the sequence $x=\left(x_{j k}\right)$ defined by

$$
x_{j k}=\left\{\begin{array}{ll}
1, & \text { if } j=n^{3}, k=m^{3} \\
0, & \text { otherwise }
\end{array} \quad n=1,2, \ldots ; m=1,2, \ldots\right.
$$


is statistically convergent of order $\tilde{\alpha}$ with $S_{\tilde{\alpha}}^{2}-\lim x_{j k}=0$ for $\tilde{\alpha}>1 / 3$ that is $s>1 / 3, t>1 / 3$ but it is not convergent.

Definition 4. Let $\widetilde{\alpha} \in(0,1]$ be given. A sequence $x=\left(x_{j k}\right)$ is said to be Cesàro summable of order $\widetilde{\alpha}$ if there is a complex number $\ell$ such that

$$
\lim _{n, m \rightarrow \infty} \frac{1}{n^{s} m^{t}} \sum_{j=1}^{n} \sum_{k=1}^{m} x_{j k}=\ell
$$

which is the case when we say that $x$ is Cesàro summable of order $\widetilde{\alpha}$ to $\ell$. For $\widetilde{\alpha} \cong 1$, the Cesàro summability of order $\widetilde{\alpha}$ reduces to the Cesàro summability that is given in [15]. The set of all Cesàro summable double sequences of order $\tilde{\alpha}$ will be denoted by $w_{\tilde{\alpha}}^{2}$. The set of all Cesàro summable double sequences will be denoted by $w^{2}$.

Definition 5. Let $\widetilde{\alpha} \in(0,1]$ be given, and let $p$ be a positive real number. Then, a sequence $x=\left(x_{j k}\right)$ is said to be strongly $p$-Cesàro summable of order $\widetilde{\alpha}$ if there is a complex number $\ell$ such that

$$
\lim _{n, m \rightarrow \infty} \frac{1}{n^{s} m^{t}} \sum_{j=1}^{n} \sum_{k=1}^{m}\left|x_{j k}-\ell\right|^{p}=0
$$

which is the case when we say that $x$ is strongly $p$-Cesàro summable of order $\widetilde{\alpha}$ to $\ell$. For $\widetilde{\alpha} \cong 1$, the strong $p$ Cesàro summability of order $\tilde{\alpha}$ reduces to the strong $p$-Cesàro summability that is given in [13]. The set of all strongly $p$ Cesàro summable sequences of order $\tilde{\alpha}$ will be denoted by $\left[w_{p}^{2}\right]_{\tilde{\alpha}}$.

\section{Main Results}

In this section, we give the main results of the paper. In Theorem 6 , we give the relationship between the statistical double convergence of order $\tilde{\alpha}$ and the statistical double convergence of order $\widetilde{\beta}$ for $\widetilde{\alpha} \preceq \widetilde{\beta}$ and so that the relationship between the statistical double convergence of order $\tilde{\alpha}$ and the statistical convergence. In Corollary 10, we give the relationship between the strong $p$-Cesàro summability of order $\widetilde{\alpha}$ and the strong $p$-Cesàro summability of order $\widetilde{\beta}$. In Theorem 12, we give the relationship between the strong $p$-Cesàro summability of order $\widetilde{\alpha}$ and the statistical double convergence of order $\widetilde{\beta}$.

Theorem 6. Let $\widetilde{\alpha}, \widetilde{\beta} \in(0,1]$ be given such that $\widetilde{\alpha} \preceq \widetilde{\beta}$. Then, $S_{\widetilde{\alpha}}^{2} \subseteq S_{\widetilde{\beta}}^{2}$ and the inclusion is strict for some $\tilde{\alpha}$ and $\widetilde{\beta}$ such that $\tilde{\alpha} \prec \tilde{\beta}$.

Proof. Let $\widetilde{\alpha}, \widetilde{\beta} \in(0,1]$ be given. If $\widetilde{\alpha} \preceq \widetilde{\beta}$ and so that $s \leq u$ and $t \leq v$, then

$$
\begin{aligned}
& \frac{1}{n^{u} m^{v}}\left|\left\{(j, k): j \leq n, k \leq m,\left|x_{j k}-\ell\right| \geq \varepsilon\right\}\right| \\
& \quad \leq \frac{1}{n^{s} m^{t}}\left|\left\{(j, k): j \leq n, k \leq m,\left|x_{j k}-\ell\right| \geq \varepsilon\right\}\right|
\end{aligned}
$$

for every $\varepsilon>0$ and this gives that $S_{\widetilde{\alpha}}^{2} \subseteq S_{\widetilde{\beta}}^{2}$. To show that the inclusion is strict, consider the sequence $x=\left(x_{j k}\right)$ defined by

$$
x_{j k}=\left\{\begin{array}{ll}
1, & j=n^{2}, k=m^{2} \\
0, & \text { otherwise }
\end{array} \quad m, n=1,2,3, \ldots\right.
$$

Then, $S_{\widetilde{\beta}}^{2}-\lim x_{k}=0$; that is, $x \in S_{\widetilde{\beta}}^{2}$ for $\widetilde{\beta} \in(1 / 2,1]$ (i.e., for $1 / 2<u \leq 1$ and $1 / 2<v \leq 1)$ but $x \notin S_{\tilde{\alpha}}^{2}$ for $\tilde{\alpha} \in(0,1 / 2]$ (i.e., for $0<s \leq 1 / 2$ and $0<t \leq 1 / 2$ ).

If we take $\widetilde{\beta} \cong 1$ in Theorem 6 , then we obtain the following result.

Corollary 7. If a double sequence is statistically convergent of order $\widetilde{\alpha}$ to $\ell$, for some $\widetilde{\alpha}$ such that $\widetilde{\alpha} \in(0,1]$, then it is statistically convergent to $\ell$; that is $S_{\widetilde{\alpha}}^{2} \subseteq S^{2}$, and the inclusion is strict.

From Theorem 6, we have the following results and the proof is easy.

Corollary 8. Let $\tilde{\alpha}, \widetilde{\beta} \in(0,1]$ be given. Then,

(i) $S_{\widetilde{\alpha}}^{2}=S_{\widetilde{\beta}}^{2}$ if and only if $\widetilde{\alpha} \cong \widetilde{\beta}$;

(ii) $S_{\widetilde{\alpha}}^{2}=S^{2}$ if and only if $\widetilde{\alpha} \cong 1$.

The proof of the following theorem is straight forward from the definition, so omitted.

Theorem 9. Let $\widetilde{\alpha}, \widetilde{\beta} \in(0,1]$ be given such that $\widetilde{\alpha} \preceq \widetilde{\beta}$, and let $p$ be a positive real number. Then, $\left[w_{p}^{2}\right]_{\tilde{\alpha}} \subseteq\left[w_{p}^{2}\right]_{\tilde{\beta}}$ and the inclusion is strict for some $\tilde{\alpha}$ and $\widetilde{\beta}$ such that $\widetilde{\alpha} \prec \widetilde{\beta}$.

Proof. Let $x=\left(x_{k}\right) \in\left[w_{p}^{2}\right]_{\widetilde{\alpha}}$. Then, given $\widetilde{\alpha}$ and $\widetilde{\beta}$ such that $\widetilde{\alpha} \preceq \widetilde{\beta}$ and a positive real number $p$, we may write

$$
\frac{1}{n^{u} m^{v}} \sum_{j=1}^{n} \sum_{k=1}^{m}\left|x_{j k}-\ell\right|^{p} \leq \frac{1}{n^{s} m^{t}} \sum_{j=1}^{n} \sum_{k=1}^{m}\left|x_{j k}-\ell\right|^{p},
$$

and this gives that $\left[w_{p}^{2}\right]_{\widetilde{\alpha}} \subseteq\left[w_{p}^{2}\right]_{\widetilde{\beta}}$.

To show that the inclusion is strict, consider the sequence $x=\left(x_{j k}\right)$ defined in (14). It is easy to see that

$$
\frac{1}{n^{u} m^{v}} \sum_{j=1}^{n} \sum_{k=1}^{m}\left|x_{j k}\right|^{p} \leq \frac{\sqrt{n} \sqrt{m}}{n^{u} m^{v}}=\frac{1}{n^{u-1 / 2} m^{v-1 / 2}} .
$$

Since $1 /\left(n^{u-1 / 2} m^{v-1 / 2}\right) \rightarrow 0$ as $n \rightarrow \infty, m \rightarrow \infty$, then $\left[w_{p}^{2}\right]_{\widetilde{\beta}}-\lim x_{j k}=0$; that is, $x \in\left[w_{p}^{2}\right]_{\widetilde{\beta}}$ for $\widetilde{\beta} \in(1 / 2,1]$ (i.e., for $1 / 2<u \leq 1$ and $1 / 2<v \leq 1$ ), but since

$$
\frac{\sqrt{n}-1}{n^{s}} \frac{\sqrt{m}-1}{m^{t}} \leq \frac{1}{n^{s} m^{t}} \sum_{j=1}^{n} \sum_{k=1}^{m}\left|x_{j k}\right|^{p}
$$


and $\left((\sqrt{n}-1) / n^{s}\right)\left((\sqrt{m}-1) / m^{t}\right) \rightarrow \infty$ as $n \rightarrow \infty, m \rightarrow \infty$, then $x \notin\left[w_{p}^{2}\right]_{\tilde{\alpha}}$ for $\widetilde{\alpha} \in(0,1 / 2]$ (i.e., for $0<s<1 / 2$ and $0<t<1 / 2)$. This completes the proof.

The following result is a consequence of Theorem 9.

Corollary 10. Let $\widetilde{\alpha}, \widetilde{\beta} \in(0,1]$ be given such that $\widetilde{\alpha} \preceq \widetilde{\beta}$, and let $p$ be a positive real number. Then,

(i) $\left[w_{p}^{2}\right]_{\widetilde{\alpha}}=\left[w_{p}^{2}\right]_{\widetilde{\beta}}$ if and only if $\widetilde{\alpha} \cong \widetilde{\beta}$;

(ii) $\left[w_{p}^{2}\right]_{\tilde{\alpha}} \subseteq w_{p}^{2}$ for each $\tilde{\alpha}$ such that $\tilde{\alpha} \in(0,1]$ and $0<$ $p<\infty$.

The following result is a simple consequence of Hölder's inequality which is an extension of a result of Maddox [27].

Theorem 11. Let $\tilde{\alpha} \in(0,1]$, and let $0<p<q<\infty$. Then, $\left[w_{q}^{2}\right]_{\tilde{\alpha}} \subset\left[w_{p}^{2}\right]_{\tilde{\alpha}}$.

Taking $\widetilde{\alpha} \cong 1$ in Theorem 11 , we obtain a result of Maddox [27]: if $0<p<q<\infty$, then $w_{q}^{2} \subset w_{p}^{2}$.

Theorem 12. Let $\widetilde{\alpha}$ and $\widetilde{\beta}$ be given such that $\widetilde{\alpha} \preceq \widetilde{\beta}$, and let $0<$ $p<\infty$, where $\widetilde{\alpha}, \widetilde{\beta} \in(0,1]$. If a sequence is strongly $p$-Cesàro summable of order $\tilde{\alpha}$ to $\ell$, then it is statistically convergent of $\operatorname{order} \tilde{\beta}$ to $\ell$.

Proof. For any sequences $x=\left(x_{j k}\right)$ and $\varepsilon>0$, we have

$$
\begin{aligned}
& \sum_{j=1}^{n} \sum_{k=1}^{m}\left|x_{j k}-\ell\right|^{p} \\
& \quad \geq\left|\left\{(j, k): j \leq n, k \leq m,\left|x_{j k}-\ell\right| \geq \varepsilon\right\}\right| \cdot \varepsilon^{p},
\end{aligned}
$$

so that since $\tilde{\alpha} \preceq \widetilde{\beta}$,

$$
\begin{aligned}
& \frac{1}{n^{s} m^{t}} \sum_{j=1}^{n} \sum_{k=1}^{m}\left|x_{j k}-\ell\right|^{p} \\
& \geq \frac{1}{n^{s} m^{t}}\left|\left\{(j, k): j \leq n, k \leq m,\left|x_{j k}-\ell\right| \geq \varepsilon\right\}\right| \cdot \varepsilon^{p} \\
& \geq \frac{1}{n^{u} m^{v}}\left|\left\{(j, k): j \leq n, k \leq m:\left|x_{j k}-\ell\right| \geq \varepsilon\right\}\right| \cdot \varepsilon^{p} .
\end{aligned}
$$

From this, it follows that if $x=\left(x_{j k}\right)$ is strongly $p$-Cesàro summable of order $\widetilde{\alpha}$ to $\ell$, then it is statistically convergent of order $\widetilde{\beta}$ to $\ell$. result.

If we take $\widetilde{\beta} \cong \widetilde{\alpha}$ in Theorem 12 , we obtain the following

Corollary 13. Let $\tilde{\alpha} \in(0,1]$ be given, and let $0<p<\infty$. If a double sequence is strongly p-Cesàro summable of order $\tilde{\alpha}$ to $\ell$, then it is statistically convergent of order $\tilde{\alpha}$ to $\ell$.

Remark 14. Note that the converse of Theorem 12 does not hold in general. We see that a bounded and statistically convergent double sequence of order $\widetilde{\alpha}$ need not be strongly $p$-Cesàro summable of order $\tilde{\alpha}$ in general for $\tilde{\alpha} \in(0,1]$.
The sequence $x=\left(x_{j k}\right)$ defined by

$$
x_{j k}=\left\{\begin{array}{ll}
\frac{1}{\sqrt{j} \sqrt{k}}, & j \neq n^{3}, k \neq m^{3} \\
1, & \text { otherwise }
\end{array} \quad m, n=1,2,3, \ldots\right.
$$

is an example for this case. It is clear that $x \in \ell_{\infty}^{2}$ and $x \in S_{\tilde{\alpha}}^{2}$ for each $\widetilde{\alpha} \in(1 / 3,1]$. First, recall that the inequality

$$
\sum_{j=1}^{n} \sum_{k=1}^{m} \frac{1}{\sqrt{j} \sqrt{k}}>\sqrt{m} \sqrt{n}
$$

holds for every positive integer $m \geq 2$ and $n \geq 2$. Define $G_{n}=\left\{j \leq n: j \neq i^{2}, i=1,2,3, \ldots\right\}, H_{m}=\left\{k \leq m: k \neq i^{2}, i=\right.$ $1,2,3, \ldots\}$ and take $p=1$. Since

$$
\begin{aligned}
& \sum_{j=1}^{n} \sum_{k=1}^{m}\left|x_{j k}\right|^{p} \\
& =\sum_{j=1}^{n} \sum_{k=1}^{m}\left|x_{j k}\right|=\sum_{j \in G_{n}, 1 \leq j \leq n} \sum_{k \in H_{m}, 1 \leq k \leq m}\left|x_{j k}\right| \\
& \quad+\sum_{j \notin G_{n}, 1 \leq j \leq n} \sum_{k \notin H_{m}, 1 \leq k \leq m}\left|x_{j k}\right| \\
& =\sum_{j \in G_{n}, 1 \leq j \leq n} \sum_{k \in H_{m}, 1 \leq k \leq m} \frac{1}{\sqrt{j} \sqrt{k}} \\
& \quad+\sum_{j \notin G_{n}, 1 \leq j \leq n} \sum_{k \notin H_{m}, 1 \leq k \leq m} 1>\sum_{j=1}^{n} \sum_{k=1}^{m} \frac{1}{\sqrt{j} \sqrt{k}}>\sqrt{m} \sqrt{n},
\end{aligned}
$$

we have

$$
\begin{aligned}
& \frac{1}{n^{s} m^{t}} \sum_{j=1}^{n} \sum_{k=1}^{m}\left|x_{j k}\right|^{p} \\
& =\frac{1}{n^{s} m^{t}} \sum_{j=1}^{n} \sum_{k=1}^{m}\left|x_{j k}\right|>\frac{1}{n^{s} m^{t}} \sum_{j=1}^{n} \sum_{k=1}^{m} \frac{1}{\sqrt{j} \sqrt{k}} \\
& >\frac{1}{n^{s} m^{t}} \sqrt{n} \sqrt{m} \\
& =\frac{1}{n^{s-1 / 2}} \cdot \frac{1}{m^{t-1 / 2}} \longrightarrow \infty \quad \text { as } m \longrightarrow \infty, n \longrightarrow \infty,
\end{aligned}
$$

so that $x \notin\left[w_{p}^{2}\right]_{\widetilde{\alpha}}$ for $\tilde{\alpha} \in(0,1 / 2]$ if $p=1$. Therefore, $x \in$ $S_{\widetilde{\alpha}}^{2}-\left[w_{p}^{2}\right]_{\widetilde{\alpha}}$ for $\widetilde{\alpha} \in(1 / 3,1 / 2]$ if $p=1$.

Corollary 15. Let $\tilde{\alpha} \in(0,1]$, and let $p$ be a positive real number. Then, $w_{p \tilde{\alpha}}^{2} \subset S^{2}$. The inclusion is strict if $\widetilde{\alpha} \in(0,1)$.

Proof. From Corollaries 13 and 7, we have $\left[w_{p}^{2}\right]_{\widetilde{\alpha}} \subset S^{2}$. To show that the inclusion is strict, consider the sequence $x=$ $\left(x_{j k}\right)$ defined in (10). Then, clearly $S^{2}-\lim x_{j k}=0$; that is, 
$x \in S^{2}$ but $x \notin\left[w_{p}^{2}\right]_{\tilde{\alpha}}$ for $\tilde{\alpha} \in(0,1 / 3]$ and $p=1$. Indeed it is easy to see that

$$
\begin{aligned}
& \frac{1}{n^{s} m^{t}} \sum_{j=1}^{n} \sum_{k=1}^{m}\left|x_{j k}-0\right|^{p} \\
& \quad=\frac{1}{n^{s} m^{t}} \sum_{j=1}^{n} \sum_{k=1}^{m}\left|x_{j k}\right| \geq \frac{\sqrt[3]{n}-1}{n^{s}} \cdot \frac{\sqrt[3]{m}-1}{m^{t}} .
\end{aligned}
$$

Since $(\sqrt[3]{n}-1) / n^{s} \rightarrow \infty$ as $n \rightarrow \infty$ and $(\sqrt[3]{m}-1) / m^{t} \rightarrow \infty$ as $m \rightarrow \infty$, then $x \notin\left[w_{p}^{2}\right]_{\tilde{\alpha}}$ if $\tilde{\alpha} \in(0,1 / 3]$ and $p=1$. Consequently, $x \in S^{2}-\left[w_{p}^{2}\right]_{\widetilde{\alpha}}$ for $\widetilde{\alpha} \in(0,1 / 3]$ and $p=1$. This completes the proof.

\section{Conclusion}

The concepts of statistical convergence and strong Cesàro summability of double sequences of complex or real numbers have been studied by various mathematicians. In this paper, we introduced the concepts of statistical convergence of order $\widetilde{\alpha}$ and strong $p$-Cesàro summability of order $\widetilde{\alpha}$ for double sequences, where $\tilde{\alpha} \in(0,1]$. These concepts are much more general than the concepts of statistical convergence and strong $p$-Cesàro summability of double sequences that include these concepts in the special case $\widetilde{\alpha} \cong 1$.

Note that the converse of Theorem 12 does not hold in general; that is, a statistically convergent sequence of order $\widetilde{\alpha}$ (even a bounded and statistically convergent sequence of order $\tilde{\alpha}$ ) need not be strongly $p$-Cesàro summable of order $\tilde{\alpha}$ in general.

\section{References}

[1] H. Steinhaus, "Sur la convergence ordinaire et la convergence asymptotique," Colloquium Mathematicum, vol. 2, pp. 73-74, 1951.

[2] H. Fast, "Sur la convergence statistique," Colloquium Mathematicum, vol. 2, pp. 241-244, 1951.

[3] I. J. Schoenberg, "The integrability of certain functions and related summability methods," The American Mathematical Monthly, vol. 66, pp. 361-375, 1959.

[4] J. A. Fridy, "On statistical convergence," Analysis, vol. 5, no. 4, pp. 301-313, 1985.

[5] J. S. Connor, "The statistical and strong $p$-Cesàro convergence of sequences," Analysis, vol. 8, no. 1-2, pp. 47-63, 1988.

[6] I. J. Maddox, "Statistical convergence in a locally convex space," Mathematical Proceedings of the Cambridge Philosophical Society, vol. 104, no. 1, pp. 141-145, 1988.

[7] D. Rath and B. C. Tripathy, "Matrix maps on sequence spaces associated with sets of integers," Indian Journal of Pure and Applied Mathematics, vol. 27, no. 2, pp. 197-206, 1996.

[8] T. Šalát, "On statistically convergent sequences of real numbers," Mathematica Slovaca, vol. 30, no. 2, pp. 139-150, 1980.

[9] B. C. Tripathy, A. Baruah, M. Et, and M. Gungor, "On almost statistical convergence of new type of generalized difference sequence of fuzzy numbers," Iranian Journal of Science and Technology Transaction A, vol. 36, no. 2, pp. 147-155, 2012.
[10] B. C. Tripathy, "On statistical convergence," Proceedings of the Estonian Academy of Sciences, vol. 47, no. 4, pp. 299-303, 1998.

[11] B. C. Tripathy and A. Baruah, "Lacunary statically convergent and lacunary strongly convergent generalized difference sequences of fuzzy real numbers," Kyungpook Mathematical Journal, vol. 50, no. 4, pp. 565-574, 2010.

[12] B. C. Tripathy, "Matrix transformation between some classes of sequences," Journal of Mathematical Analysis and Applications, vol. 206, no. 2, pp. 448-450, 1997.

[13] Mursaleen and O. H. H. Edely, "Statistical convergence of double sequences," Journal of Mathematical Analysis and Applications, vol. 288, no. 1, pp. 223-231, 2003.

[14] V. Karakaya, N. Şimşek, M. Ertürk, and F. Gürsoy, " $\lambda$-statistical convergence of sequences of functions in intuitionistic fuzzy normed spaces," Journal of Function Spaces and Applications, vol. 2012, Article ID 926193, 14 pages, 2012.

[15] F. Móricz, "Statistical convergence of multiple sequences," Archiv der Mathematik, vol. 81, no. 1, pp. 82-89, 2003.

[16] B. C. Tripathy and A. J. Dutta, "Bounded variation double sequence space of fuzzy real numbers," Computers \& Mathematics with Applications, vol. 59, no. 2, pp. 1031-1037, 2010.

[17] B. C. Tripathy and B. Sarma, "Some double sequence spaces of fuzzy numbers defined by Orlicz functions," Acta Mathematica Scientia B, vol. 31, no. 1, pp. 134-140, 2011.

[18] B. C. Tripathy and B. Sarma, "Statistically convergent difference double sequence spaces," Acta Mathematica Sinica, vol. 24, no. 5, pp. 737-742, 2008.

[19] S. Bhunia, P. Das, and S. K. Pal, "Restricting statistical convergence," Acta Mathematica Hungarica, vol. 134, no. 1-2, pp. 153161, 2012.

[20] R. Çolak, "Statistical convergence of double sequences of order $(\alpha, \beta)$," in Proceedings of the 4th Congress of the Turkic World Mathematical Society (TWMS '11), Baku, Azerbaijan, July 2011.

[21] S. A. Mohiuddine, A. Alotaibi, and M. Mursaleen, "Statistical convergence of double sequences in locally solid Riesz spaces," Abstract and Applied Analysis, vol. 2012, Article ID 719729, 9 pages, 2012.

[22] S. A. Mohiuddine, H. Sevli, and M. Cancan, "Statistical convergence of double sequences in fuzzy normed spaces," Filomat, vol. 26, no. 4, pp. 673-681, 2012.

[23] A. D. Gadjiev and C. Orhan, "Some approximation theorems via statistical convergence," The Rocky Mountain Journal of Mathematics, vol. 32, no. 1, pp. 129-138, 2002.

[24] R. Çolak, "Statistical convergence of order $\alpha$," in Modern Methods in Analysis and Its Applications, pp. 121-129, Anamaya Publishers, New Delhi, India, 2010.

[25] R. Çolak and Ç. A. Bektaş, " $\lambda$-statistical convergence of order $\alpha$," Acta Mathematica Scientia B, vol. 31, no. 3, pp. 953-959, 2011.

[26] A. Pringsheim, "Zur Theorie der zweifach unendlichen Zahlenfolgen," Mathematische Annalen, vol. 53, no. 3, pp. 289-321, 1900.

[27] I. J. Maddox, "Spaces of strongly summable sequences," The Quarterly Journal of Mathematics, vol. 18, no. 1, pp. 345-355, 1967. 


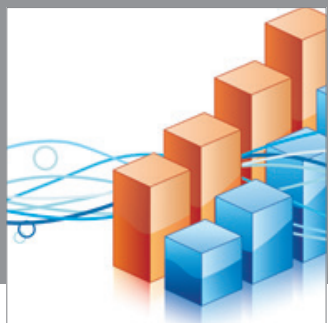

Advances in

Operations Research

mansans

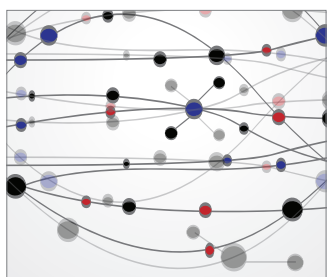

The Scientific World Journal
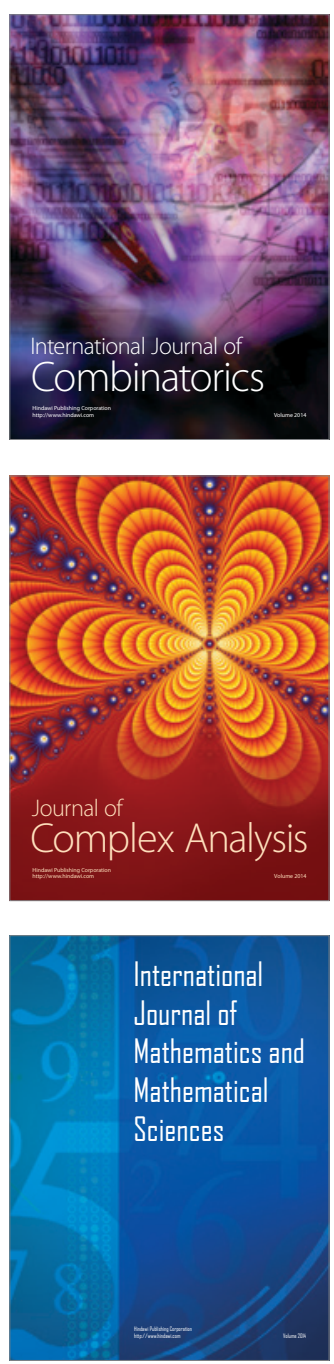
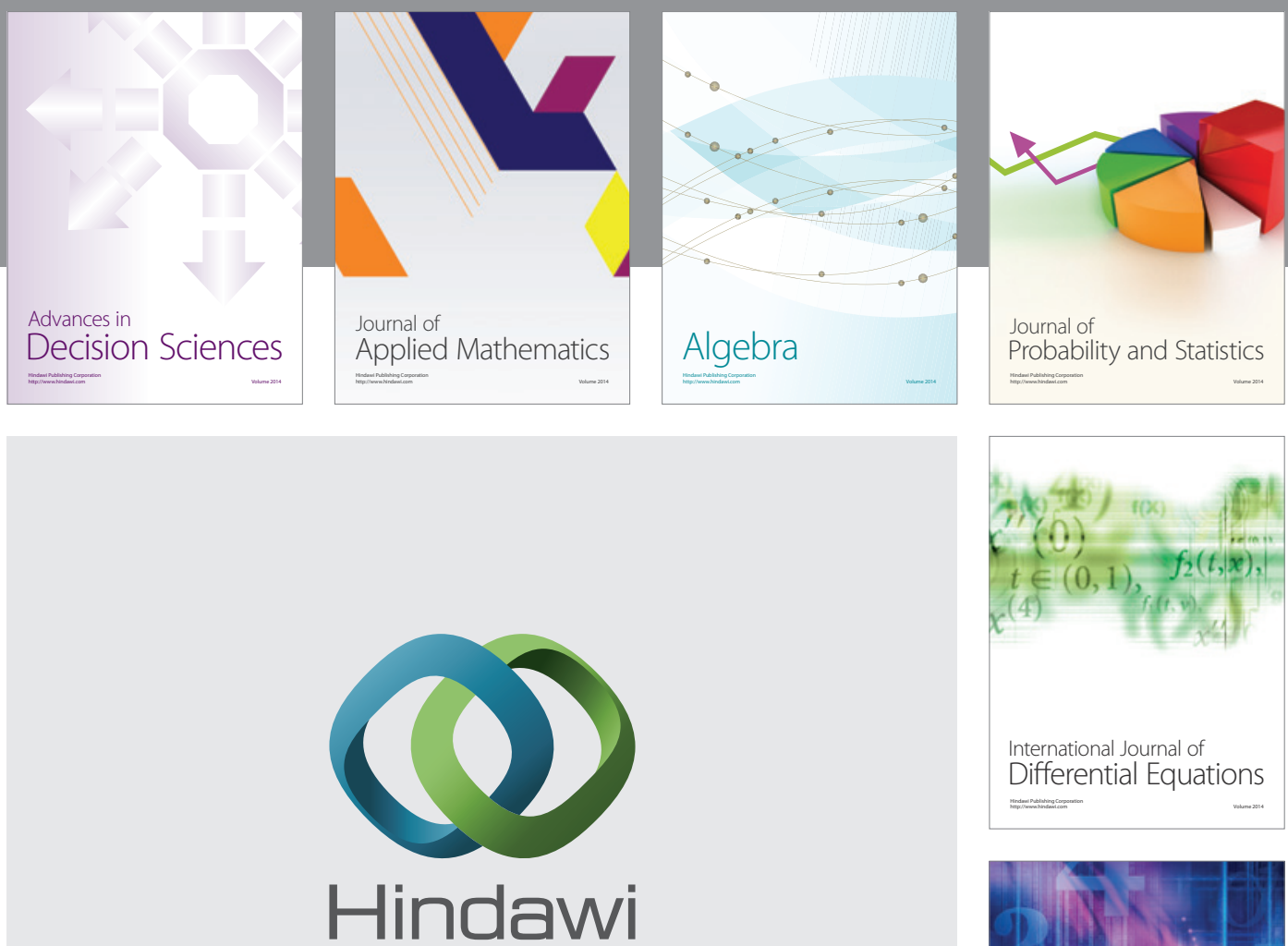

Submit your manuscripts at http://www.hindawi.com
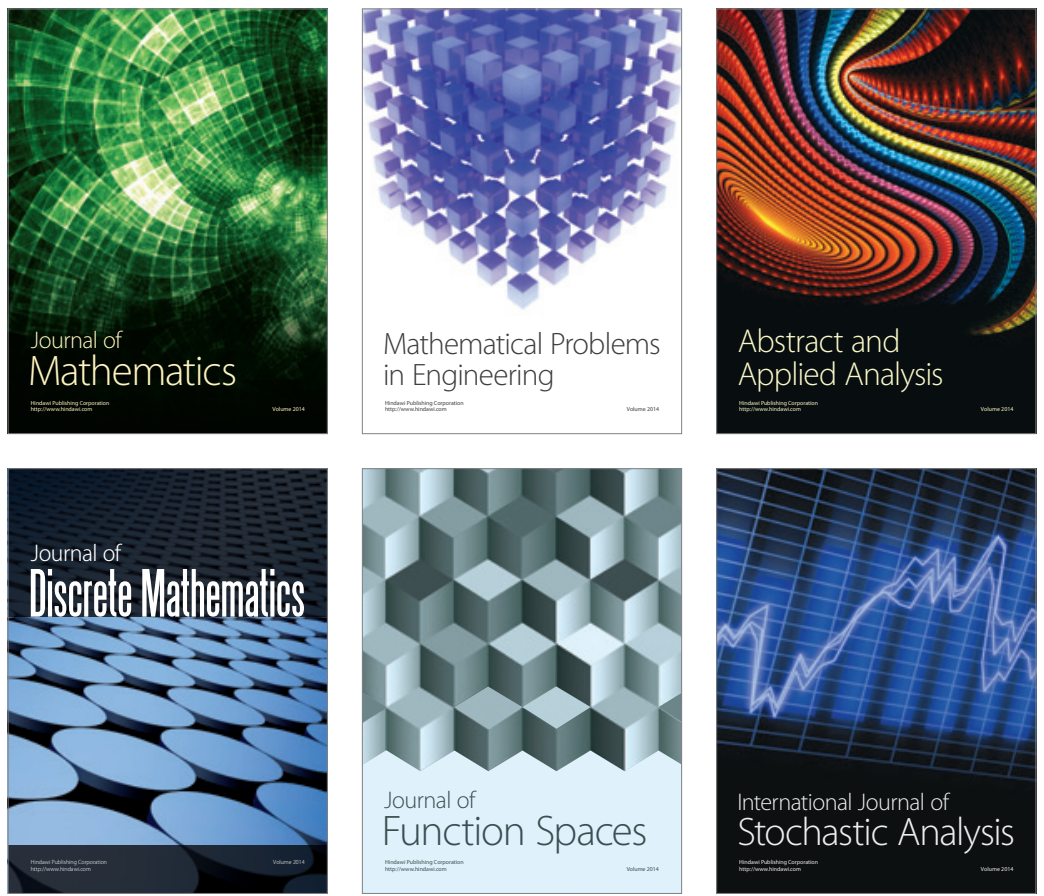

Journal of

Function Spaces

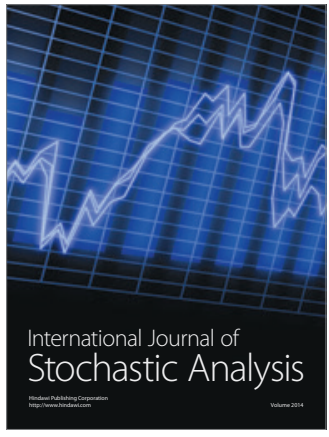

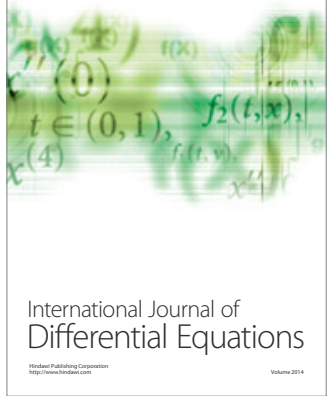
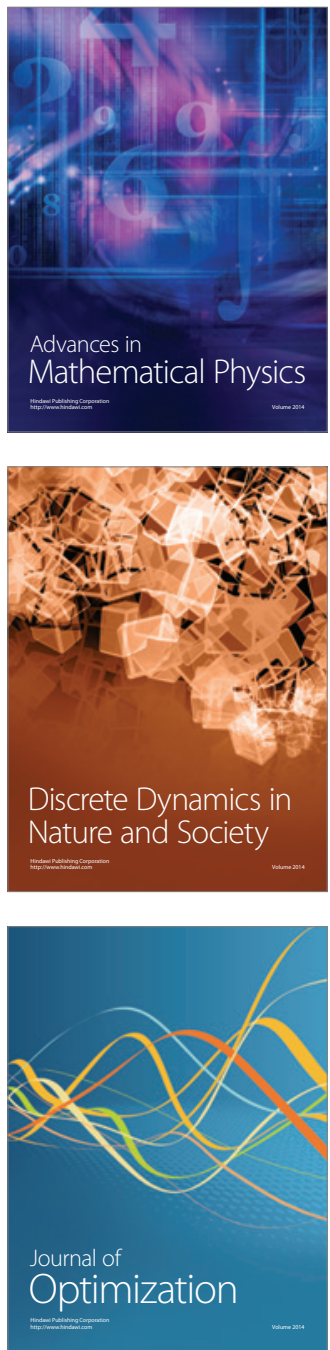